\title{
Evaluación de la contribución de los bonos de carbono a la competitividad de las centrales solares de concentración en Chile
}

\author{
Assessment of the contribution of carbon credits for the competitiveness of \\ concentrating solar power plants in Chile
}

Ricardo T. Rivas $^{1} \quad$ José M. Cardemil ${ }^{1}$

Recibido 18 de junio de 2014, aceptado 8 de enero de 2015

Received: June 18, 2014 Accepted: January 8, 2015

\begin{abstract}
RESUMEN
En Chile el $68 \%$ de la energía eléctrica consumida es generada por centrales térmicas accionadas por combustibles fósiles, los que son importados en $90 \%$. Las variaciones en el precio de los combustibles afectan directamente al sector industrial, lo que podría convertirse en un inconveniente para el desarrollo del país. Las energías renovables pueden ser una respuesta a la preocupación por la seguridad energética y el cambio climático. Sin embargo, su despliegue avanza a una velocidad menor a la deseable debido a la alta percepción de riesgo que se les asigna a estos proyectos. El presente trabajo estima la contribución de los bonos de carbono a la competitividad de las centrales solares de concentración. Para ello utiliza simulaciones de plantas solares realizadas con el software SAM y cálculos de reducción de emisiones con metodologías oficiales de la UNFCCC. Estos factores de emisión son proyectados al futuro mediante diferentes escenarios energéticos para determinar los aumentos de la competitividad de la energía solar que genera la particularidad climática del país. Esta contribución argumenta que la percepción de riesgo es equivocada y que es necesario evaluar los flujos en el largo plazo, ya que fuentes de energía como la solar de concentración han demostrado ser competitivas bajo las condiciones adecuadas de clima, financiamiento e incentivos. Adicionalmente, se ha observado que ingresos por bonos de carbono pueden representar una baja considerable en los Costos Nivelados de Energía (LCOE) de las tecnologías solares de concentración.
\end{abstract}

Palabras clave: Energía solar, competitividad, bonos de carbono, matriz energética.

\begin{abstract}
Currently in Chile about $68 \%$ of the electricity is generated by thermal power plants, consuming fossil fuels which are approximately $90 \%$ imported. The high variations on the price of such resources affect directly the industrial production, and therefore hindering the national development. Nonetheless, Chile presents high availability of renewable energy sources, such as solar and wind, which can represent a viable alternative in achieving the challenges in energy security and climate change mitigation goals. Despite that, the current deployment of renewable energy technologies in Chile is not significant, mainly due to the high risk associated to such projects. The aim of the present work is to assess the contribution of the carbon credits to the competitiveness of concentrating solar power plants. The evaluation is carried out by several simulations using the System Advisor Model software, coupled with the official methodologies from UNFCCC for estimating the emission reduction and considering several scenarios for the composition of the Chilean electricity matrix. Through this analysis it is possible to establish that the risk perception is not justified, since at the particular climatic conditions of northern Chile, can be competitive with other energy sources. In this context, the key variables are the solar irradiation,
\end{abstract}

\footnotetext{
1 Escuela de Ingeniería Industrial. Universidad Diego Portales. Av. Ejército 441. Santiago, Chile. E-mail: tomasrivas00@gmail.com; jose.cardemil@udp.cl
} 
financing alternatives and tax incentives. However, the carbon credits can also represent a decrease of the Levelized Cost of Energy (LCOE), depending on the evolution of the carbon market.

Keywords: Solar energy, competitiveness, carbon credits, energy matrix.

\section{INTRODUCCIÓN}

Chile cuenta con un amplio rango de recursos naturales como cobre, molibdeno, yodo y celulosa. La producción, extracción, agregación de valor y exportaciones de estos recursos le ha permitido al país ser considerado de ingreso alto por el banco mundial y ser invitado a formar parte de la OCDE. Sin embargo, esta nación explota limitadamente sus recursos energéticos propios y posee una casi nula producción de combustibles fósiles [1].

La actividad minera e industrial del país requiere de altas cantidades de energía eléctrica, lo que equivale al $64 \%$ del total de esta energía generada en Chile [1]. La electricidad en el país es generada en un $68 \%$ por plantas termoeléctricas accionadas por combustibles fósiles, como se muestra en la Figura 1. Sin embargo, en la región norte de Chile, donde se concentran gran cantidad de faenas mineras, la energía eléctrica es generada en $99 \%$ con estos combustibles [3], los que son importados en $90 \%$ [1].

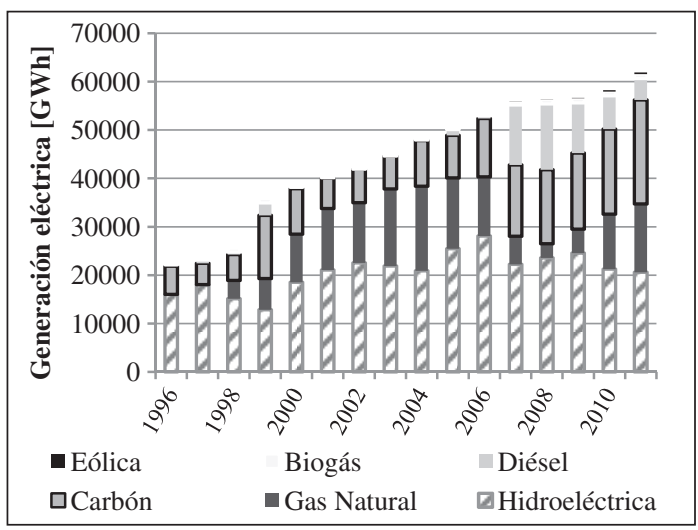

Figura 1. Histórico de generación eléctrica SIC y SING (GWh).

Fuente: CADE [2].

Una alternativa para hacer frente a la fuerte dependencia energética de Chile son las Energías Renovables No Convencionales (ERNC), las que se definen como aquellas cuya fuente de energía primaria es biomasa, hidráulica menor a $20 \mathrm{MW}$, geotérmica, solar, eólica, mareas, olas, o gradiente térmico. Estas fuentes energéticas permiten aprovechar la alta disponibilidad de recursos autóctonos del país, y cuentan con mayor aprobación de la ciudadanía que otras energías como las termoeléctricas. Actualmente las ERNC representan solo 5,9\% de la generación total de energía eléctrica. De este total, $30 \%$ de energía eólica, $30 \%$ de energía hidráulica de pequeña escala, $40 \%$ de biomasa y un discreto $0,5 \%$ de energía solar [4].

No obstante la baja participación de la energía solar, actualmente existen proyectos solares aprobados por la autoridad ambiental que ascienden a $3 \mathrm{GW}$ y existe otro GW adicional en tramitación [3]. La escasa puesta en marcha de los proyectos de energía solar aprobados se debe, entre otros factores, a su alto costo de instalación y la tendencia de los inversionistas de evitar el riesgo en inversiones en países en desarrollo donde no existen datos de recurso solar confiables y debidamente detallados. En adición a lo último, existe una limitada exploración de los ingresos potenciales por bonos de carbono (BC) [5].

Los ingresos adicionales por BC, como también las favorables condiciones climáticas de Chile, podrían significar aumentos en la competitividad de las centrales solares de concentración, debido a que ambos factores aportan a la disminución de sus costos nivelados de energía. Lo que sería capaz de cambiar la percepción de riesgo financiero actual asociada a la energía solar, logrando mejores condiciones de financiamiento y en consecuencia mayor despliegue de estas tecnologías.

En la literatura técnica, algunas publicaciones internacionales analizan la competitividad de las tecnologías solares y consideran el efecto de los bonos de carbono, pero solo para el caso de aplicaciones fotovoltaicas [6-7]. Entretanto, Andrade y Pérez [8] presentaron un análisis de BC para la realidad chilena, incluyendo varias ERNC. Pero el 
trabajo no presenta cálculos propios del factor de emisión ni consideran la metodología adecuada. La generación de las plantas es estimada en promedios mundiales, por lo que no considera las externalidades y otros beneficios de instalar las plantas en Chile. Finalmente, no presenta proyecciones futuras del factor de emisión, lo que limita sus resultados, ya que los ingresos por $\mathrm{BC}$ varían año a año, debido a la composición de la matriz energética y efectos de mercado.

Olave [9] presentó una evaluación completa de las tecnologías solares. Sin embargo, no realizó simulaciones y consideró datos de radiación que provienen de una base de datos no validada con mediciones en terreno [10], lo que se traduce en incertidumbre en el cálculo de los BC.

Recientemente, Greenhill [11] presentó una revisión exhaustiva del mercado del carbono en Chile, pero esta recopilación de información no contiene cálculos numéricos, ni análisis de tecnologías de generación de energía en particular. Esto último no permite la evaluación de la contribución de los BC. Por otro lado, el trabajo de Del Sol y Sauma [12] analiza estimaciones de los impactos económicos de instalar plantas solares en el norte de Chile. Pero utilizan datos climáticos que no han sido validados con datos superficiales. Además, se considera un precio fijo para los bonos de carbono según el valor del año 2010 (US\$18). Este precio es impensado hasta en los escenarios más optimistas para 2014 de US\$ 10 [13]. Los ingresos por venta de bonos de carbono son calculados solo para un año y replicados para el cálculo de los años siguientes, lo que no considera variaciones del factor de emisión en una matriz energética cambiante.

Según el conocimiento de los autores no existen otros trabajos que analicen el aporte de los bonos de carbono a la competitividad de las plantas de energía solar de concentración en Chile. Este país es de interés para la evaluación de tecnologías solares debido a que posee uno de los mejores climas del planeta para la implementación de esta energía [14]. El presente trabajo pretende entregar una visión precisa y robusta sobre esta problemática, puesto que la componente económica es el principal determinante en el proceso de decisión de inversión en proyectos de energía solar, junto con un correspondiente análisis de riesgo.
Para estimar la generación eléctrica de plantas solares en Chile y aumentos en su competitividad se consideran configuraciones de plantas solares existentes en España y Estados Unidos. Estas plantas son simuladas en territorio chileno utilizando el software de simulación System Advisor Model (SAM) [15]. De esta manera se estima el desempeño que tendrían de instalarse en localidades representativas en Chile. La herramienta SAM es utilizada en varias publicaciones científicas y además sus creadores han validado sus simulaciones con datos reales de plantas existentes. Así, el presente trabajo se diferencia de las investigaciones anteriores principalmente porque utiliza metodologías consolidadas por la Convención Marco de las Naciones Unidas (UNFCCC) para cálculos de reducciones de emisiones resultantes de un proyecto de energía solar. Adicionalmente cuenta con escenarios energéticos proyectados hasta el año 2030. Para ello se consideran los resultados publicados en Escenarios Energéticos 2030 [16]. Esto permite evaluar los Certificados de Reducción de Emisiones (CER) en un mercado spot y no reducir los ingresos aceptando contratos futuros que asignan primas por riesgo.

Adicionalmente, este trabajo considera escenarios de precios de bonos de carbono actualizados, los que permiten tener una apreciación actual de los beneficios que entrega el protocolo de Kioto a los proyectos que presentan reducciones de emisiones. En este artículo se destaca la calidad de los datos de radiación solar utilizados en las simulaciones, los que fueron obtenidos del Atlas Solar de Chile [14]. Estos datos presentan incertidumbres conocidas de aproximadamente $10 \%$, mientras que los otros trabajos antes mencionados sobre localidades de Chile utilizan datos de NASA, NREL o Meteonorm, los que no han sido validados con estaciones superficiales.

Los costos por unidad de energía eléctrica generada por una planta solar dependen directamente de la radiación solar, por tanto, estos costos son diferentes en cada localidad. El objetivo de este trabajo es determinar precisamente los costos de la energía solar en ciertas localidades de Chile, incluyendo el posible aumento de competitividad que significa la venta de bonos de carbono y compararlos con tecnologías convencionales de generación de potencia. Por último, parte de esta metodología puede ser utilizada para el cálculo de reducción 
de emisiones futuras para cualquier proyecto de generación de energía que sea considerado de cero emisiones, como es el caso de las ERNC.

\section{Mecanismo de desarrollo limpio}

El protocolo de Kioto es un acuerdo internacional entre 193 países cuyo fin último es la reducción de emisiones de gases de efecto invernadero (GEI) a escala global, el que fue recientemente revalidado hasta el año 2020 [17, 18]. Este protocolo cuenta con tres mecanismos para cumplir con su objetivo, de los que el presente trabajo utiliza Mecanismo de Desarrollo Limpio (MDL), por ser el que se aplica al caso chileno. Este mecanismo permite a países en desarrollo comercializar las reducciones de emisiones resultantes de proyectos desarrollados dentro del mismo país. Para la comercialización es necesaria la certificación de esta reducción de emisiones por la UNFCCC. Esta entidad entrega al desarrollador del proyecto un CER por cada tonelada métrica de $\mathrm{CO}_{2}$ que se dejó de emitir debido a la operación del proyecto. Los CER son adquiridos por países desarrollados y utilizados para cumplir con los compromisos de reducción de emisiones estipulados en el protocolo de Kioto.

Con la idea de unificar la unidad de medida para cada uno de los GEI se crea la unidad dióxido de carbono equivalente $\left(\mathrm{CO}_{2} \mathrm{e}\right)$, que expresa el potencial de calentamiento global que presentan otros gases, comparados al potencial del $\mathrm{CO}_{2}$ (Tabla 1).

$$
1 \text { ton de } \mathrm{CO}_{2} e=1 \mathrm{CER}=1 \mathrm{BC}
$$

Tabla 1. Equivalencias GEI.

\begin{tabular}{|ll|r|}
\hline \multicolumn{2}{|c|}{ GEI } & \multicolumn{1}{|c|}{$\mathbf{C O}_{2} \mathbf{E}$} \\
\hline Dióxido de Carbono & $\mathrm{CO}_{2}$ & $\mathbf{1}$ \\
\hline Metano & $\mathrm{CH}_{4}$ & $\mathbf{2 1}$ \\
\hline Óxido Nitroso & $\mathrm{N}_{2} \mathrm{O}$ & $\mathbf{3 1 0}$ \\
\hline Hidrofluorocarbonos & $\mathrm{HFC}$ & $\mathbf{7 4 0}$ \\
\hline Perfluorocarbonos & $\mathrm{PFC}$ & $\mathbf{1 3 0 0}$ \\
\hline Hexafluoruro de azufre & $\mathrm{SF}_{6}$ & $\mathbf{2 3 9 0 0}$ \\
\hline
\end{tabular}

\section{Escenarios energéticos 2030}

Para evaluar la contribución que significa la comercialización de los CER a la competitividad de la energía solar en Chile, es necesario conocer la composición de la matriz de generación de energía eléctrica y el crecimiento de la demanda de esta energía para los años próximos. De esta manera, es posible estimar las emisiones asociadas a la operación de los sistemas interconectados en el futuro.

Para establecer diferentes escenarios de composición futura de la matriz energética del país se consideran los resultados presentados en Escenarios Energéticos 2030 [16]. Los resultados corresponden a escenarios obtenidos por medio de la herramienta de simulación PET, que permite evaluar decisiones técnicoeconómicas sobre un universo de centrales candidatas para construcción. De esta manera, se estima la composición de la matriz energética para el año 2030 (Figura 2). Estas simulaciones consideran tanto al Sistema Interconectado Central (SIC) como al Sistema Interconectado del Norte Grande (SING).

Utilizando el criterio que han tenido hasta hoy los inversionistas del sector eléctrico, se definen los escenarios Mercado-SIC (e1), Mercado-SING (e2) y Mercado-SIC sin HidroSur (e5), este último no considera la implementación de hidroeléctricas en la región de Aysén. Los escenarios Mercado-SIC con metas ERNC (e6) y Mercado-SING con impuesto al $\mathrm{CO}_{2}$ (e7) utilizan el mismo criterio de decisiones de inversionistas, pero el primero considera un $20 \%$ de ERNC al 2020 y un $30 \%$ al 2030, mientras que el segundo considera la aplicación de impuestos a las emisiones. Por su parte, los escenarios ERNC-SIC (e3) y ERNC-SING (e4) son escenarios optimistas en la penetración de las ERNC.

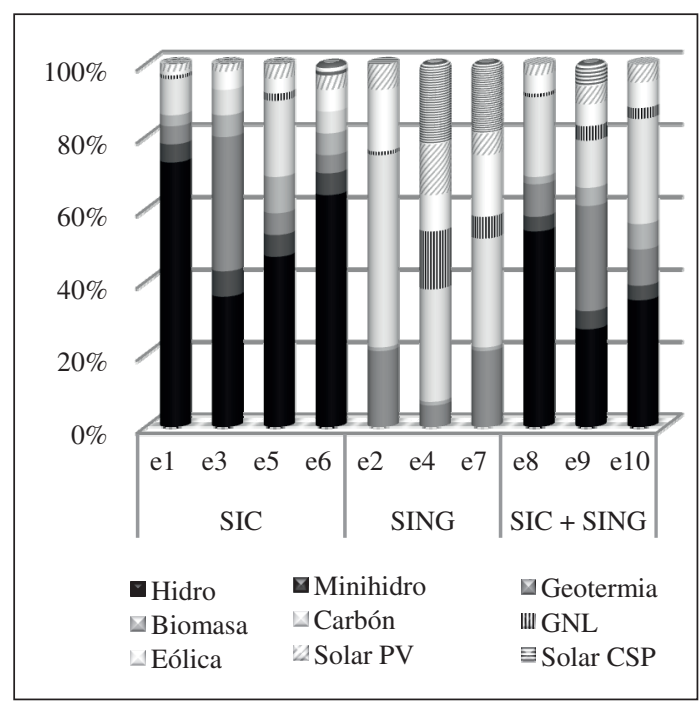

Figura 2. Composición de la matriz según tecnología, para cada escenario energético.

Fuente: EE2030 [16]. 
La interconexión SIC y SING es un tema que ha sido evaluado por expertos de la Comisión Nacional de Energía [19]. Por la posibilidad de materialización de esta medida, se definen tres escenarios de interconexión desarrollados por el autor. Mercado-SIC + Mercado-SING (e8), ERNCSIC + ERNC-SING (e9), Mercado-SIC-s/HidroSur + Mercado-SING (10).

\section{METODOLOGÍA DE EVALUACIÓN}

El factor de emisión de una tecnología de generación de energía eléctrica es un indicador que entrega información sobre la cantidad de $\mathrm{CO}_{2}$ emitido por unidad de energía generada. Para el caso de la energía eléctrica se expresa en $\mathrm{tCO}_{2} / \mathrm{MWh}$. La estimación de este factor para cada sistema interconectado y para cada año futuro, es necesaria para determinar la reducción de emisiones resultantes de la operación de una planta solar.

\section{Proyección de escenarios energéticos}

Debido a que los resultados de [16] solo entregan escenarios para el año 2030, para calcular el factor de emisión anual es necesario realizar proyecciones de los sistemas interconectados de generación de energía eléctrica desde el año 2012 al 2030. Lo anterior, por simplicidad, se lleva a cabo mediante proyecciones lineales de los porcentajes de participación de cada una de las tecnologías de generación en ambas matrices energéticas.

El crecimiento de demanda de energía eléctrica también debe ser considerado en estos escenarios, por esta razón, el presente trabajo utiliza proyecciones estimadas en [16] conforme muestra la Figura 3.

\section{Plantas solares de concentración}

La reducción de emisiones de una planta de energía renovable está correlacionada con la cantidad de energía eléctrica que esta produce. Así, mientras mayor sea su producción de energía, menor será la necesidad de utilizar combustibles fósiles para satisfacer la demanda eléctrica.

Para estimar la cantidad de energía eléctrica que genera un proyecto de energía solar se realizan simulaciones en el software SAM [15]. Estas simulaciones consideran tecnologías solares de concentración, debido a que su naturaleza térmica permite la utilización de almacenamiento de energía,

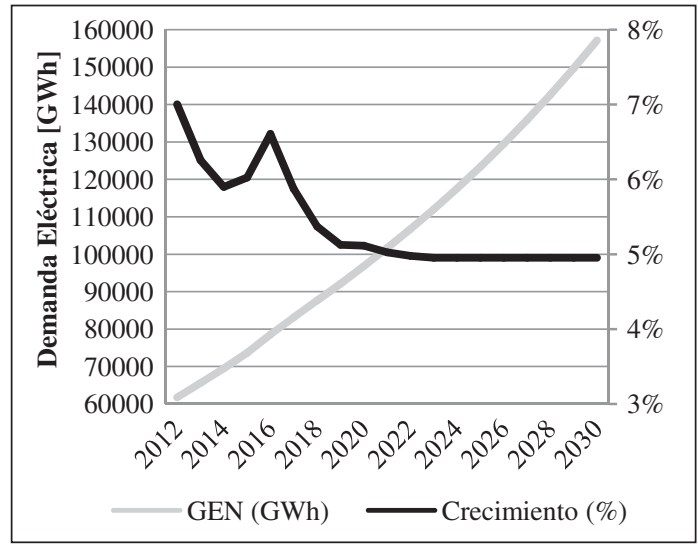

Figura 3. Proyección de la Demanda eléctrica. Fuente: EE2030 [16], modificado.

dando estabilidad al sistema y aumentando su factor de planta. Esto último facilita su operación incluso en la ausencia del sol, por lo que estas tecnologías serían la alternativa más adecuada para satisfacer los requerimientos energéticos constantes de las operaciones mineras del norte del país.

Las simulaciones consideran como referencia plantas solares en operación actualmente en España y Estados Unidos, cuyas potencias de diseño se muestran en la Tabla 2, mientras que las especificaciones técnicas y datos de operación son declarados en [20]. Algunas de estas especificaciones son altura de torre, cantidad de heliostatos o tipo de receptor. Asimismo, los datos climáticos utilizados en las simulaciones son obtenidos a partir de los resultados de [14] y resumidos en la Tabla 3.

Tabla 2. Características de las plantas analizadas.

\begin{tabular}{|l|l|c|}
\hline \multicolumn{1}{|c|}{ Planta } & \multicolumn{1}{c|}{ Tecnología } & Potencia (MW) \\
\hline Andasol 1 & C. Parabólico & 50 \\
\hline Solana & C. Parabólico & 280 \\
\hline Gemasolar & Torre & 19,9 \\
\hline Cresent Dunes & Torre & 110 \\
\hline
\end{tabular}

Tabla 3. Promedio anual de la radiación directa normal en las localidades analizadas.

\begin{tabular}{|l|c|}
\hline Localidad & $\begin{array}{c}\text { Irradiación directa } \\
\text { normal }\left(\mathbf{k W h} / \mathbf{m}^{2}\right)\end{array}$ \\
\hline Curicó & 1677 \\
\hline Santiago & 2537 \\
\hline D. Almagro & 3041 \\
\hline Calama & 3606 \\
\hline
\end{tabular}


La simulación de las plantas solares en cada una de las localidades entrega una visión sobre el comportamiento de estas plantas en el caso de que estas operaran bajo las condiciones chilenas. Al comparar los datos de radiación presentados en la Tabla 3, entre las localidades chilenas y las localidades en donde las plantas están construidas, se aprecia que la radiación en Chile es sustancialmente superior [14]. Esto indica que en este territorio las plantas solares producirían mayor cantidad de energía, y consecuentemente, una mayor cantidad de BC podría ser comercializado. De esta forma estas condiciones permiten una reducción sustancial en los costos de generación.

\section{Factor de emisión}

Para determinar el factor de emisión de un sistema eléctrico, se utiliza la metodología ACM0002 [21, 22]. El factor de emisión se evalúa por medio del cálculo de un factor de emisiones de Margen Combinado (CM) de un sistema eléctrico.

El factor de emisión de margen combinado es el resultado del promedio balanceado de factores de emisión. El Margen de Operación (OM), que se refiere a las emisiones de las plantas existentes que van a ser afectadas por el proyecto MDL y el Margen de Construcción (BM), que se refiere al grupo de plantas prospectos cuya construcción y futura operación va a ser afectada por el proyecto MDL. El margen combinado se calcula conforme a la siguiente ecuación:

$$
\begin{gathered}
E F_{\text {grid }, C M, y}=E F_{\text {grid }, O M, y} \\
+E F_{\text {grid }, B M, y} \omega_{O M}
\end{gathered}
$$

donde $E F_{\text {grid,CM,y }}$ es el factor de emisión del margen combinado ( $\left.\mathrm{tCO}_{2} / \mathrm{MWh}\right), E F_{\text {grid,BM,y }}$ es el factor de emisión del margen de construcción, $E F_{\text {grid,OM,y }}$ es el factor de emisión del margen de operación, $\omega_{O M} \mathrm{y}$ $\omega_{B M}$ son lo pesos asignados a cada factor de emisión en porcentaje. Para el caso particular de la energía solar, estos factores asumen valores numéricos de 0,75 y 0,25 respectivamente, según sugiere [21]. La norma define como plantas Low Cost (LC) como aquellas con bajo costo marginal o despachadas independiente de la carga diaria o estacional de la red [22]. Estas incluyen hidroeléctricas, centrales de pasada, geotérmicas, eólicas, biomasa de bajo costo, nucleares y solares.

\section{Margen de operación}

El factor de emisión del margen de operación se expresa conforme a la siguiente ecuación:

$$
\begin{aligned}
& E F_{\text {grid }, O M-a d j, y} \\
& =\left(1-\lambda_{y}\right) \frac{\sum_{m} E G_{m, y} E F_{E L, m, y}}{\sum_{m} E G_{m, y}} \\
& +\lambda_{y} \frac{\sum_{k} E G_{k, y} E F_{E L, k, y}}{\sum_{k} E G_{k, y}}
\end{aligned}
$$

donde $\mathrm{EF}_{\text {grid,OM-adj,y }}$ es el factor de emisión de $\mathrm{CO}_{2}$ del margen de operación simple ajustado en el año $y$, en $\mathrm{tCO}_{2} / \mathrm{MWh}, \lambda_{y}$ es un ponderador de información sobre la generación Low Cost en el año y, el que será explicado en extenso posteriormente. Mientras que $E G_{m, y}$ es la cantidad neta de electricidad generada y entregada a la red por la planta $m$ en el año $y$, $E G_{k, y}$ es la cantidad neta de electricidad generada y entregada a la red por la planta $k$ en el año $y$. Los factores de emisión para cada planta son expresos por $E F_{E L, m, y}$ y $E F_{E L, k, y}$. El subíndice $m$ corresponde a todas las plantas sirviendo a la red en el año y excepto las de bajo costo, finalmente, el subíndice $k$ corresponde a todas las plantas de bajo costo sirviendo a la red en el año $y$.

El SING cuenta con casi nulas fuentes de energías renovables, por lo que el ponderador $\lambda_{y}$ es considerado cero, de acuerdo como indica la norma. Para el caso del SIC es necesario calcular el factor $\lambda_{y}$ para cada escenario energético y cada año comprendido en la evaluación del proyecto.

El factor de emisión de cada planta $\left(E F_{E L, m, y}\right)$ cuenta con dos ecuaciones para su cálculo, el uso de ellas depende de la cantidad de información disponible. Cuando se cuenta con información sobre el tipo de combustible, la cantidad utilizada y los MWh de generación de energía eléctrica, se calcula conforme a la siguiente ecuación:

$$
E F_{E L, m, y}=\frac{\sum_{m} F C_{i, m, y} N C V_{i, y} E F_{C O 2, i, y}}{E G_{m, y}}
$$

donde $E F_{E L, m, y}$ es el factor de emisión de una planta $m$ en el año $y$ ( $\mathrm{tCO}_{2} / \mathrm{MWh}$ ), $F C_{i, m, y}$ es la cantidad de combustible $i$ consumido por la planta $m$ en el año 
y. $N C V_{i, y}$ es el valor calorífico neto (contenido de energía) del tipo de combustible $i$ en el año $y$ (GJ/ unidad de masa o volumen), $E G_{m, y}$ es la cantidad neta de electricidad generada y despachada a la red por la planta $m$ en el año $y$. Finalmente, $m$ son todas las plantas no Low Cost.

Entretanto, cuando falta información sobre la cantidad de combustible utilizada y solo se cuenta con el tipo de combustible y la generación de la planta, la ecuación señalada por la norma es:

$$
E F_{E L, m, y}=\frac{E F_{C O 2, m, i, y} \cdot 3,6}{\eta_{m, y}}
$$

donde $E F_{C O 2, m, i, y}$ es el factor de emisión promedio del combustible $i$ usado por la planta $m$ en el año $y, 3,6$ es un factor de ponderación definido por la UNFCCC y $\eta_{m, y}$ es la eficiencia de conversión de energía promedio. Finalmente, $m$ son todas las plantas no-Low Cost.

Para el cálculo de $E F_{E L, m, y}$, tanto para el SIC, como para el SING, se considera la ecuación (5). Lo anterior debido a que los cálculos son basados en proyecciones futuras, por lo que se utilizan factores de emisión por defecto para cada tecnología obtenidos del Panel Intergubernamental para el Cambio Climático [23]. Las plantas de bajo costo son consideradas de emisión cero $\left(E F_{E L, k, y}=0\right)$.

El ponderador $\lambda_{y}$ es un indicador de la porción de la generación de energía que corresponde a fuentes renovables en una matriz energética. A medida que este ponderador aumenta su valor, disminuye el factor de emisión de operación $\left(E F_{O M}\right)$. En todos los escenarios presentados en la figura 2 la matriz migra gradualmente hacia las energías renovables, consideradas Low Cost por la UNFCCC. Eso último hace necesario un cálculo de $\lambda_{y}$ para cada año incluido en el análisis, lo que se realiza mediante la siguiente ecuación:

$$
\lambda_{y}=\frac{\text { horas low cost en el margen }}{8760 \text { horas del año }}
$$

Para determinar las horas en que las plantas Low Cost están en el margen de operación, es necesario realizar el siguiente procedimiento: Los datos cronológicos de carga en MWh para cada hora del año, se ordenan de mayor a menor, con lo que se obtiene la denominada curva de duración, según se observa en la Figura 4.

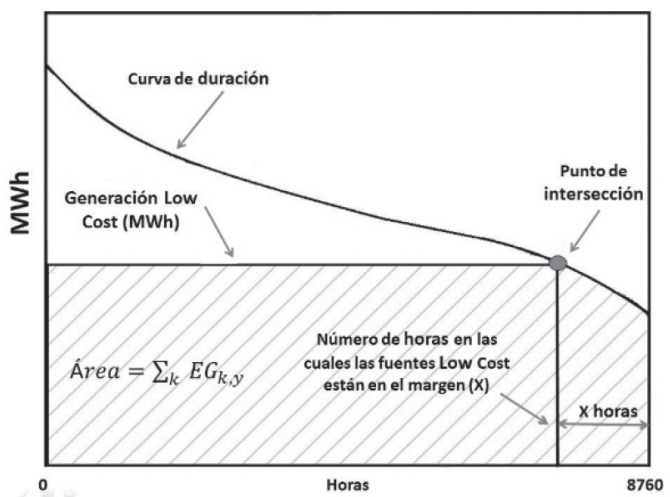

Figura 4. Curva de generación horaria ordenada por cantidad de energía generada.

Fuente: AM0002 modificado [22].

Por su parte la información de generación en MWh de las centrales Low Cost se grafica formando una línea horizontal mediante la curva de duración, tal que el área de la región inferior a la línea horizontal y la curva sea igual a la generación en MWh de las plantas Low Cost. Posteriormente, se localiza la intersección de la línea horizontal graficada y la curva de duración, siendo este punto el que indica el número de horas del año en que las plantas Low Cost están en el margen o son capaces de satisfacer la demanda eléctrica por si solas. Si las líneas no se intersectan, se puede concluir que las plantas Low Cost no aparecen en el margen y que $\lambda_{y}$ es igual cero.

La generación horaria para los años futuros corresponde a la generación horaria actual incrementada con los porcentajes de crecimiento de demanda proyectados en [16], conforme a la Figura 3.

\section{Margen de construcción}

Este factor de emisión del margen de construcción refleja el efecto de una matriz energética cambiante y se calcula con el último $20 \%$ de plantas construidas. Por simplicidad, y debido a la incertidumbre sobre las plantas a construir en el futuro, es que el presente trabajo calcula este factor de emisión como una relación entre el $E F_{B M}$ actual y el porcentaje de plantas Low Cost para cada año incluido en el análisis. De esta manera, $E F_{B M}$ entrega información sobre las plantas por construirse y no pierde su objetivo de reflejar cambios en la matriz energética. 
Una vez determinados $E F_{\text {grid,OM-adj,y }}$ y $E F_{\text {grid,BM,y, }}$, es posible utilizar la ecuación (2) para determinar el factor de emisión de margen combinado de la red $\left(E F_{\text {grid,CM,y }}\right)$, para cada año del horizonte del análisis.

\section{Reducción de emisiones}

La cantidad de generación anual de energía eléctrica (MWh) de las plantas solares concentradoras obtenida de las simulaciones, junto a los factores de emisión proyectados, permiten estimar la reducción anual de emisiones de la siguiente manera:

$$
\begin{array}{r}
t \mathrm{CO}_{2}(t / a \tilde{n} o)=E F\left(t C \mathrm{O}_{2} / \mathrm{MWh}\right) \\
\cdot E(M W h / a \tilde{n} o)
\end{array}
$$

donde $t \mathrm{CO}_{2}$ representa la reducción de $\mathrm{CO}_{2}$ resultante de la operación de un proyecto de energía solar, $E F$ es el factor de emisión del sistema interconectado en el que se instalaría el proyecto de energía solar y $E$ representa la cantidad de energía eléctrica generada por la planta. Al multiplicar año tras año la generación de una planta solar por el factor de emisión correspondiente al escenario energético a evaluar, se obtiene la reducción de emisiones resultante de la operación de cada planta solar.

Una vez determinada la reducción de emisión resultante de la operación de las plantas solares en cada año proyectado, es posible estimar el valor económico de esta reducción de emisiones. Esto se realiza considerando tres distintos escenarios de precios de BC definidos por la Bolsa del Clima de Santiago (SCX). Además, se considera un cuarto escenario con un precio de los BC correspondiente al inicio del protocolo de Kioto, condición que podría darse si Europa recupera su fuerza económica. Estos escenarios son resumidos en la Tabla 4.

Tabla 4. Escenarios de precio de BC.

\begin{tabular}{|l|c|}
\hline \multicolumn{1}{|c|}{ Escenario } & Valor de los BC (US\$ ) \\
\hline Pesimista & $\mathbf{4}$ \\
\hline Medio & $\mathbf{6 , 5}$ \\
\hline Optimista & $\mathbf{1 2}$ \\
\hline Inicial & $\mathbf{2 6}$ \\
\hline
\end{tabular}

Fuente: Bolsa del Clima de Santiago.

Una vez estimado el valor económico (en dólares) que representa la comercialización de la reducción de emisiones para cada uno de los escenarios, plantas y localidades, es posible evaluar el aporte que este ingreso adicional representa para los proyectos.

\section{Costos nivelados de energía}

La competitividad de las plantas analizadas se evalúa en términos de los Costos Nivelados de la Energía (LCOE), que corresponden a los costos de generación por cada unidad de energía producida. Los LCOE son la manera más utilizada en publicaciones científicas para comparar tecnologías de generación de electricidad. Este indicador no depende del precio de la energía eléctrica, ni de políticas de tarificación para energías renovables. La independencia de variables propias de la localidad donde se instala una planta o variaciones de mercado, valida a los LCOE como una forma adecuada para comparar plantas de generación de electricidad [24]. Respecto de lo expresado anteriormente, los LCOE se calculan según la ecuación (8):

$$
L C O E=\frac{I_{0}+\sum_{t=1}^{n} \frac{A_{t}+B C_{t}}{(1+i)^{t}}}{\sum_{t=1}^{n} \frac{E_{t}}{(1+i)^{t}}}
$$

donde LCOE son los costos nivelados de energía en US\$ $/ \mathrm{kWh}, I_{0}$ representa el monto de inversión inicial, $A_{t}$ los costos anuales de operación, $B C_{t}$ son los ingresos por Bonos de Carbono, $E_{t}$ es la cantidad de electricidad anual que genera la planta, $i$ es la tasa de descuento, $n$ es el horizonte de tiempo en la evaluación del proyecto y $t$ el año de operación. La ecuación (8) se diferencia de la expresión convencional de LCOE, debido a que considera ingresos por la comercialización de BC.

Los costos de operación y la generación de energía eléctrica de las plantas solares fueron estimados mediante las simulaciones descritas en la sección anterior. Es necesario mencionar que las proyecciones más lejanas del factor de emisión son hasta el año 2030, mientras que, las simulaciones de las plantas solares consideran una vida útil de 25 años, hasta el 2037. El factor de emisión considerado para los últimos siete años de evaluación es considerado constante.

La Figura 5 muestra los LCOE promedio de diferentes tecnologías de generación eléctrica a modo de referencia. Se observa que las plantas solares 
de concentración presentan LCOE tres o cuatro veces superiores al promedio de otras tecnologías de generación.

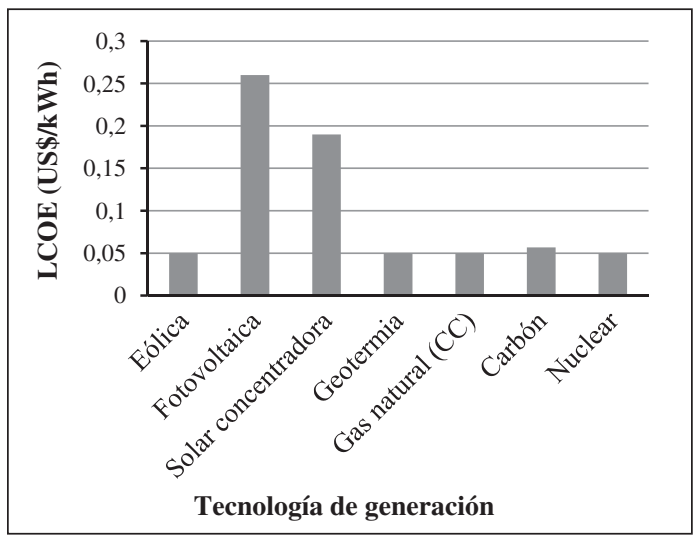

Figura 5. LCOE promedio de diferentes tecnologías de generación eléctrica.

Fuente: NREL [24].

\section{RESULTADOS}

Las simulaciones realizadas indican que de instalarse una central equivalente a Andasol 1 en la localidad de Calama, bajo las mismas condiciones de financiamiento, la central bajaría sus costos nivelados de energía hasta en un $37 \%$ en comparación con la central original instalada en España. Esto se debe solo al aumento en los niveles de radiación solar, sin considerar ingresos por BC. Esto último valida al territorio chileno como un lugar de interés para el estudio de la competitividad de las tecnologías solares de concentración.

Existen tres factores que pueden incidir en los costos de las plantas solares de concentración instaladas en Chile. El primero es la composición de la matriz energética, ya que en una matriz con alta participación de energías renovables, el factor de emisión de un sistema interconectado es considerablemente menor. Esto último se traduce en una menor reducción de emisiones por parte de la planta solar y consecuentemente un menor ingreso por BC.

El segundo factor es la radiación solar disponible en la localidad de la planta de generación, donde los diferentes índices de radiación significan mayor generación de energía y en consecuencia menores costos LCOE. El tercer factor es el precio de los
$\mathrm{BC}$, debido a que los ingresos por comercialización de $\mathrm{BC}$ de una planta dependen directamente de su valor de mercado.

La Figura 6 muestra el efecto de la radiación solar en los costos nivelados de energía. La composición de la matriz energética considerada para el análisis fue la del escenario e1 para plantas ubicadas en el SIC y e2 para plantas ubicadas en el SING, el precio de BC considerado es de US\$12. Como se observa en esta figura, los LCOE pueden disminuir hasta en 54\% de una localidad a otra. Esto último evidencia la relevancia de la calidad de los datos de radiación para el estudio de las tecnologías solares. La incertidumbre en estos datos representa cambios significativos en la evaluación de los proyectos de estas plantas generadoras, lo que finalmente se traduce en inversionistas con información inadecuada para la toma de decisiones. Por otro lado, la figura 6 resalta lo significativa que resulta la correcta selección de localidad para la instalación de una planta solar, debido a lo determinante que demuestra ser este factor, tanto para la correcta evaluación financiera de estos proyectos, como para la competitividad de esta tecnología.

La Figura 6 evidencia también que las plantas solares de concentración de torre (en azul) presentan economías de escala, lo que permite a una planta solar como Cresent Dunes (100 MW) reducir su LCOE en hasta $21 \%$ respecto de una planta de torre de menor tamaño como Gemasolar (20 MW). Esta economía de escala podría deberse

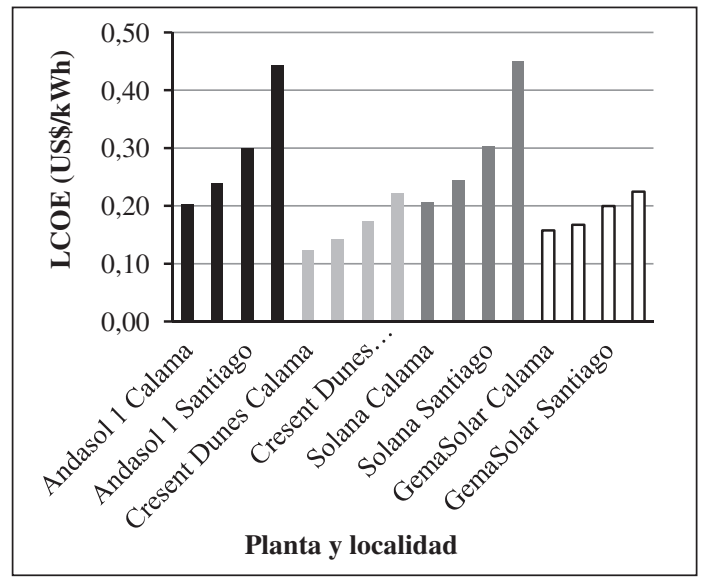

Figura 6. LCOE de las plantas estudiadas según localidad de instalación. 
a la particularmente baja humedad ambiente de la localidad de Calama. Esta condición climática logra reducir en parte la atenuación de los rayos solares debido a la absorción de radiación que ocurre a bajos niveles de la atmósfera [25]. Por otra parte, este resultado se podría atribuir a la tecnología utilizada para el almacenamiento térmico de las plantas solares, ya que ambas plantas cuentan con diferencias técnicas en la implementación. Este resultado entrega información que debe ser considerada por desarrolladores de proyectos para el diseño de las plantas solares, debido a que su apropiado diseño significa aumentos en la producción de energía, lo que disminuye sus costos LCOE, y finalmente conlleva cambios significativos en el valor económico del proyecto.

El efecto que causa la composición de la matriz energética futura en los LCOE, debido a los diferentes factores de emisión, fue evaluado considerando el valor de BC en US\$12, las localidades son Calama para el SING y Santiago para el SIC. Los diferentes escenarios de la matriz energética probaron que, por sí solos, no son capaces de producir cambios considerables en los LCOE, debido a que solo se encontraron aportes a su disminución en 1,4\% (US\$ 12). Por otro lado, considerando el valor de BC en US\$ 26 se encontraron aportes a la disminución de los LCOE de hasta un 3,4\%.

Estos resultados fueron obtenidos evaluando proyecciones de la matriz energética drásticamente diferentes, lo que indica, que sin importar la composición futura de la matriz energética chilena, las variaciones en el factor de emisión resultantes de los distintos escenarios no reportan cambios demasiado significativos a la competitividad de las centrales solares.

El mayor LCOE promedio de las tecnologías térmicas tradicionales de generación evaluadas con datos históricos de los años 2005 a 2012, corresponde al carbón, en centrales de ciclo combinado de gasificación integrada con US\$ 0,11 por $\mathrm{kWh}$ [24]. Por lo que este valor es considerado como referencia para determinar la competitividad de las centrales solares.

El efecto del precio de los bonos de carbono en los LCOE fue evaluado considerando la localidad de Calama y el escenario energético e2. Como se observa en la Figura 7 en los primeros tres escenarios de precios de $\mathrm{BC}$, es posible obtener diferencias cercanas al 7\% en los LCOE, respecto de un escenario sin BC. Por otra parte, al comparar el escenario de US\$26 con el escenario sin BC, se observan variaciones de hasta un $15 \%$ en los LCOE. Por lo que los BC contribuyen a la disminución de los LCOE de las centrales solares, y mientras mayor sea su precio, más interesante es esta contribución.

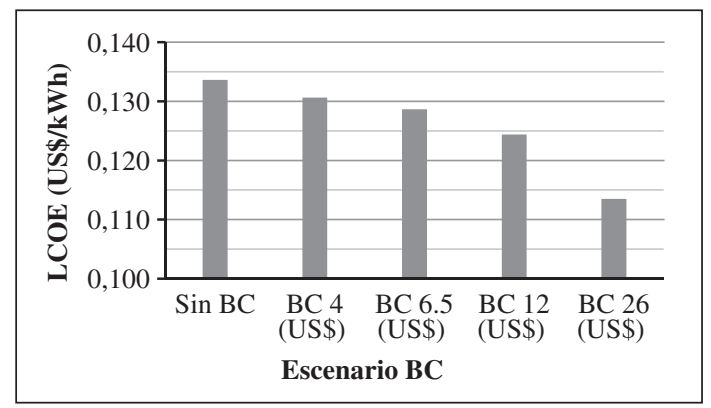

Figura 7. LCOE según escenario de BC, de una planta solar equivalente a Cresent Dunes en Calama.

Con el objetivo de cuantificar el efecto que tiene la tasa de descuento en los LCOE, y la importancia de la correcta asignación de riesgo, se realiza un análisis de sensibilidad con tres tasas diferentes. A modo de referencia, NREL evalúa estos proyectos con una tasa de 7\% [24] y la Agencia Internacional de Energía (IEA) realiza sus análisis de sensibilidad con tasas de descuento entre 5\% y $10 \%$ [26]. Además IEA realiza sus evaluaciones de proyectos solares de concentración con tasa 8\% [27]. La Figura 8 muestra los resultados del análisis de sensibilidad con relación a la tasa de descuento, para el caso

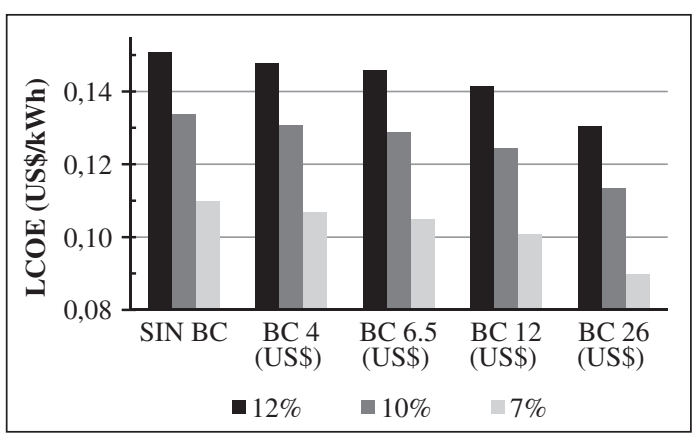

Figura 8. LCOE según valor de la tasa de descuento, de una planta equivalente a Cresent Dunes en Calama. 
particular de la central solar Cresent Dunes, simulada en la localidad de Calama, con el precio de los BC fijo en US\$ 12. Este escenario de precio de BC es el más cercano a las proyecciones para 2014 [13].

Según el conocimiento de los autores, las tasas de descuento mencionadas anteriormente son adecuadas para la realidad chilena, debido a que proyectos solares evaluados en este país cuentan con tasas del mismo orden de magnitud. Las tasas consideradas en análisis son de 7\%, 10\% y $12 \%$. La primera ha sido utilizada para la evaluación de proyectos solares pequeños con ayuda estatal, la segunda se ha utilizado para evaluar proyectos de mayor escala y la tercera tasa representa el máximo considerado para aportes estatales en el financiamiento de proyectos.

Los LCOE de las centrales solares de concentración podrían, sin problemas, llegar a niveles competitivos con una tasa de descuento de un $7 \%$ y BC en US\$ 12 con un LCOE en US\$ 0,1 por kWh. Asimismo, en un escenario más exigente, con una tasa de descuento de $10 \%$ y BC en US\$12, los costos de la energía por kWh son de US\$ 0,1244 , lo que podría considerarse competitivo, debido su cercanía con el costo por kWh de una central de carbón de ciclo combinado de gasificación. Lo anterior es evaluado sin ningún tipo de política de fomento a la generación de energías renovables, ni tampoco tasas especiales de préstamos, las que podrían ser capaces de llevar los LCOE a niveles competitivos. Las centrales solares de concentración de torre están cerca de ser competitivas por si solas, sin más ayuda que las particulares condiciones de radiación de algunas localidades chilenas.

Los cálculos de costos realizados en este trabajo no consideran opciones de financiamiento, esto con el objetivo de comparar las tecnologías en igualdad de condiciones. Sin embargo, la inversión inicial asociada a una planta solar es más elevada que en otras tecnologías y juega un rol superlativo en la evaluación de los proyectos, subiendo considerablemente los LCOE. Es en este factor donde se podrían situar de mejor manera incentivos políticos para la disminución de la percepción de riesgo de los inversionistas. Si se logra reconocer la importancia de un crecimiento estratégico de la matriz eléctrica y de contar con una matriz energética menos dependiente de combustibles extranjeros, se podrían diseñar políticas de incentivo para la realización de este tipo de proyectos solares.

\section{CONCLUSIONES}

Este trabajo representa un aporte a la desmitificación de la energía solar como una alternativa de generación de energía financieramente riesgosa y difícil de predecir, ya que en la actualidad se cuenta con datos de radiación validados en terreno con incertidumbre conocida. Los resultados encontrados para cada uno de los factores que afectan a los costos de la energía son concluyentes respecto de la competitividad de estas tecnologías.

Los escenarios energéticos futuros de la matriz energética probaron no causar mayores efectos en los cálculos de los LCOE, en su análisis de sensibilidad no consiguen generar cambios mayores al 3,4\% en los LCOE. Esto se debe a que los cambios del factor de emisión de los sistemas interconectados que estos escenarios representan, afectan solo a los ingresos por BC, los que representan una influencia moderada en los LCOE en comparación con otros factores.

Los ingresos por $\mathrm{BC}$ probaron disminuir el costo nivelado de energía de las tecnologías solares, esto según su precio de mercado. Para un precio de US\$ 12 se observaron disminuciones de los LCOE de hasta un $7 \%$. Por otra parte, para un precio de US\$ 26 los BC logran disminuciones de hasta un $15 \%$, ambos representan valores considerables.

Las condiciones climáticas también probaron ser un factor determinante para la disminución de los LCOE, puesto que permiten aumentar la producción de electricidad, lo que disminuye los LCOE. En las diferentes localidades evaluadas existen diferencias de hasta un 54\% en los costos de la energía de una localidad a otra. Lo anterior indica la importancia de la ubicación de una planta solar y establece al desierto de Atacama como un lugar idóneo a nivel mundial debido a los excelentes índices de radiación registrados y la baja humedad del aire.

Gracias a las particulares condiciones de radiación y humedad del aire de la ciudad de Calama se observa que existen rendimientos de escala para las plantas solares de concentración de torre, llegando a existir diferencias de hasta un $21 \%$ en los LCOE de una planta a otra. 
Para el caso particular de la central Cresent Dunes simulada en la localidad de Calama, los LCOE probaron ser competitivos, para una tasa de descuento de $7 \%$ y BC a un precio de US\$12. Sin embargo, para una tasa de descuento de $10 \%$ y BC en US\$12, los LCOE son de US\$ 0,1244/kWh lo cual es superior a los US $\$ 0,11 / \mathrm{kWh}$ de la tecnología a carbón mencionada anteriormente. Para alcanzar la línea de la competitividad en el caso más realista, el precio de los BC debería ser como mínimo de US\$ 25 o que las tecnologías solares cuenten con algún tipo de ayuda económica por parte del Estado.

El limitado despliegue de las tecnologías solares se atribuye a la elevada inversión inicial que esta requiere y a la falta de financiamiento adecuado. Estas dificultades de financiamiento son causadas principalmente por la alta percepción de riesgo financiero y falta de información en general.

El presente trabajo demuestra que las tecnologías solares de concentración están muy cerca de ser consideradas competitivas para condiciones realistas de mercado. Con un pequeño incentivo por parte del Estado y si se logra que el conocimiento sobre estas fuentes de energía se masifique, los inversionistas estarán informados apropiadamente de sus ventajas. Finalmente, será posible que se realicen grandes proyectos de concentración solar en un futuro cercano. Esto último porque existe la factibilidad técnica y económica para su implementación.

\section{REFERENCIAS}

[1] División de prospectiva y Política Energética del Ministerio de Energía. "Balance nacional de energía 2012”. Mayo 2013. URL: http://www.minenergia.cl

[2] CADE. "Informe anual Comisión Nacional para el Desarrollo Eléctrico". Julio 2011. URL: http://www.minenergia.cl

[3] Comisión Nacional de Energía. "Estadísticas eléctricas". Marzo 2013. URL: http://www.cne.cl/energias

[4] Centro de Energías Renovables. "Reporte CER Febrero". Febrero 2014. URL: http://cer.gob.cl

[5] F.R. Martins, E.B. Pereira, S.A. Silva, S.L. Abreu and S. Colle. "Solar energy scenarios in Brazil, part one: Resource assessment". Energy Policy. Vol. 36, Issue 8, pp. 2853-2864. 2008.
[6] K. Branker, M. Phatak and J.Pearce. "A review of solar photovoltaic levelized cost of electricity". Renewable and Sustainable Energy Reviews. Vol. 15, Issue 9, pp. 44704482. 2011.

[7] S. Reichelstein and M. Yorston. "The prospects for cost competitive solar PV power". Energy Policy. Vol. 55, pp. 117-127. 2013.

[8] A. Andrade y A. Pérez. "Análisis de los bonos de carbono: un incentivo económico para las empresas chilenas que invierten en proyectos de MDL". Memoria de Título. Universidad Austral de Chile. Noviembre 2009.

[9] D. Olave. "Evaluación técnico económica de la instalación de centrales termosolares en Chile". Memoria de título. Universidad de Talca, Diciembre 2011.

[10] Departamento de Geofísica de la Universidad de Chile. "Modelación del recurso solar y eólico en el norte de Chile". Comisión Nacional de Energía. Santiago, Chile. Mayo 2009.

[11] K. Greenhill. "Estudio del mercado del carbono en Chile". Tesis para obtener el grado de Magíster. Universidad de Chile. Junio 2013.

[12] F. del Sol and E. Sauma. "Economic impacts of installing solar power plants in northern Chile". Renewable and Sustainable Energy Reviews. Vol. 19. Issue 1, pp. 489-498. 2013.

[13] Bloomberg. "Value of the world's carbon markets to rise again in 2014". January, 2014. URL: http://about.bnef.com/press-releases/ valueof-the-worlds-carbon-markets-to-riseagain-in-2014/

[14] R. Escobar, A. Ortega, J.M. Cardemil, A. Pino y C. Cortés. "Atlas Solar de Chile". Ed. Pontificia Universidad Católica. Santiago, Chile. 2014.

[15] NREL. "System Advisor Model, v.2013.1.15". Diciembre 2013. URL: http://sam.nrel.gov

[16] Comité técnico de la plataforma Escenarios Energéticos. "Escenarios energéticos Chile 2030”. Julio 2013. URL: http://www.escenariosenergeticos.cl

[17] UNFCCC. "Nations take 'essential' next step in climate change fight". December, 2012. URL: http://www.unfecc.com

[18] UNFCCC. "Protocolo de Kyoto". Noviembre 2013. URL: http://www.unfecc.com 
[19] Comisión Nacional de Energía. "Impacto económico y social de interconexión SICSING". Junio 2013. URL: www.cne.cl

[20] NREL. "Concentrating solar projects". December, 2013. URL: www.nrel.gov/csp/ solarpaces

[21] UNFCCC. "Grid-connected electricity generation from renewable sources". July, 2008. URL: http://cdm.unfccc.int

[22] UNFCCC. "Tool to calculate the emission factor for an electricity system". November, 2013 URL: http://cdm.unfecc.int/ methodologies

[23] IPCC. "IPCC guidelines for national greenhouse gas inventories". June, 2006. URL: http://www.ipcenggip.iges.or.jp/ public/2006gl/vol1.html
[24] NREL. "Transparent cost database". November, 2013. URL:

http://www.nrel.gov/analysis/tech_lcoe.html

[25] JM. Cardemil, AR. Starke, V. Scariot and S. Colle. "Evaluating solar radiation attenuation models to assess the effects of climate and geographical location on the heliostat field efficiency". Energy Procedia, Vol. 49, pp. 1288-1297. 2014.

[26] International Energy Agency. "Projected cost of generating electricity". June, 2010. URL: http://www.iea.org

[27] International Energy Agency. "Renewable energy essentials: concentrating solar thermal power". June, 2009. URL: http://about.bnef. $\mathrm{com} /$ press-releases/valueof-the-worldscarbon-markets-to-rise-again-in-2014/ 